\title{
Thermal Image Processing Approach to Detect Malaria using Fuzzy Logic
}

\author{
Amey Walke \\ VIT University \\ Vellore
}

\author{
Goutam Ghosh \\ VIT University \\ Vellore
}

\author{
Shashikant Dewangan \\ VIT University \\ Vellore
}

\begin{abstract}
The thermal image processing technique for detecting malaria using General Fuzzy Min-Max neural network (GFMM). For detecting malaria, image should go through 4 standard steps, pre-processing, segmentation, feature extraction and selection and classification. Median filter is used in pre-processing step which reduces salt-and-pepper noise of the image. The filtered image is then segmented with the help of Otsu thresholding technique which automatically computes the optimum threshold partitioning the two classes such that spreading is minimal. The features of the segmented part are extracted by Gray Level Co-occurrence Matrix (GLCM), which extracts the infected part of the malaria blood cell. This matrix holds data of gray values of every pixel at its corresponding location. Finally, the GFMM is performed on the extracted data for classification. It performs classification along with clustering, which provides efficient way in recognizing and searching the infected part of the cell.
\end{abstract}

\section{General Terms}

Image pre-processing, Segmentation, Feature extraction.

\section{Keywords}

Image pre-processing, Median filtering, Segmentation, Otsu Thresholding, Feature extraction, GLCM, Classification, General Fuzzy Min-Max neural network.

\section{INTRODUCTION}

Malaria is a life- menacing infectious disease with universal impact: more than 198 million cases every year and ongoing malaria spreading in about 97 countries [20]. The figures publicized by World Health Organization (WHO) on malaria is tremendous. Below there are some numbers that are appropriate to illuminate the earnest of this disease:

i. $\quad 3.2$ billion (half of the world population), among which 1.2 billion are at extreme danger

ii. More than one malaria case occurs every 1100 population in high risk areas.

iii. $\quad 91 \%$ of all malaria deaths occur in sub-Saharan Africa.

iv. $\quad 79 \%$ occur in children under five.

Regardless of the possibility that intestinal sickness is a totally avoidable and treatable mosquito-borne infection in western nations, in creating nations this condition is likely because of a rare subsidizing to battle jungle fever. However in a decade ago, some development has been made, jungle fever frequency rates have been reduced by $47 \%$ all inclusive, and by 55\% in the World Health Organization African Region. These inclusions rescued roughly 4.1 million lives among which $97 \%$ are in the under-five age bunch in sub-Saharan Africa.
The criticalness in growing new analytic methodologies is animated because of this worldwide issue, since jungle fever is frequently under-analyzed and over-cured [2]. Quick and exact conclusion is essential to block grimness and mortality avoiding the peril of expanding medication resistance because of dubious treatment. Till date, in intestinal sickness analysis the finest standard remains the examination of re-colored blood movies by optical microscopy [3].Sadly, there is high rate of beguiling analytic results because of denied research center conditions in provincial nations, results in despicable treatment and to jungle fever overspread. Exact analysis takes generous time and just experienced all around gifted microscopist can diminish mistakes [3]. Especially for the deadly Plasmodium falciparum (P.f), the speedy lifesaving treatment needs high touchy conclusion and quick access to the test outcomes. In this manner, in provincial nations a symptomatic instrument for intestinal sickness must be quick, exact, simple to utilize, minimal effort and effectively represented [1].The method in which the parasite advancements convinces modifications of the host red platelet (RBC) film and cytoskeleton is exceptionally perplexing, in light of the fact that greater than 95 proteins of the RBC can experience changes with critical impact on the features of the influenced RBC and on fever pathogenesis. Dynamic strength of the cell layer is connected with the parasite development $[5,6]$. The biomechanical features of the uninfected RBCs existing in parasitized blood are additionally changed, yet the procedure is still vague [7-9].To figure biomechanical rotations of the cells various methods have been as of late advanced. These include micro fluidics, photograph acoustic and optical procedures. Micro fluidics is grounded on current improvement in creation of small scale channels with properties similar to that of the veins $[10,11]$. Utilizing hemodynamic effects as the Fahraeus effect or margination, plasma split and white-blood-cell improvement have been shown $[12,13]$.

The Margi country impact, taking into account the deformability of RBCs, has likewise been utilized to isolate contaminated RBCs from non-tainted cells in intestinal sickness [14]. Interestingly, the detachment impact is greatest in $40 \%$ hematocrit, permitting handling an expansive number of cells/unit of time. On the other hand, this procedure is restricted to $90 \%$ recuperation for late stage (thropozoite) contaminated RBCs and $75 \%$ recuperation for right on time stage (ring) tainted RBCs. The execution of the methodology of [15] as far as exactness, is still a long way from that of Giemsa strategy yet it can be utilized as a preparatory stride to build the centralization of contaminated cells in a given blood test. 


\section{LITERATURE SURVEY}

Malaria is one of the transmittable disease can also be lifethreatening in worst cases. The under developed countries are at greater risk. World Health Organization (WHO) in its report says that malaria influences roughly a huge number of deaths and more than 225 million infections every year. The illness is emerged by parasites fitting in with the sort Plasmodium, of which Plasmodium falciparum gives $97 \%$ of deaths. The conclusion of the contaminations because of $\mathrm{P}$. falciparum is still completed by means of manual systems particularly in developing nations. Despite the fact that there are progressed systems for finding, manual microscopy of blood films on slides is still thought to be the highest quality level. Manual microscopy has advantage over different methods in that it is both delicate and particular. One of the weaknesses of determination utilizing manual microscopy techniques is that it requires broad human mediation amid the demonstrative procedure which can frequently prompt late and some of the time wrong findings. The microscopist requires broad preparing to pick up mastery in the conclusion, and as a result of the sheer volume of the specimens that should be analyzed; the strategy is not steady and is reliant upon blood spread and stain quality, magnifying instrument quality and the ability of the microscopist. The past study, outlines a self-loader recognizable proof methodology subject to picture preparing and one that offered a tried and true and stable result. The writing contains depictions and points of interest of a few computer-vision or picture-based calculations. Be that as it may, the vast majority of these calculations are directed and complex, that is they require manual mediation or adjustment. Considering the high casualty rate and gigantic volumes of tests that should be examined we require a delicate, handy and vigorous strategy with least human intercession. In this setting, computer-based conclusion can help in the quick, exact and predictable identification of genuine malaria cases, guaranteeing that just those patients with malaria are dealt with. Manual microscopy is completed by inspecting slim blood films on slides under the magnifying lens and reporting the rate of parasitaemia (i.e. number of contaminated red platelets (iRBCs) for more than 100 infinitesimal fields). Microscopists likewise need to distinguish parasite morphology by different life cycle stages for speciation, portrayed in The WHO viable microscopy guide. Giemsa re-coloring is most broadly used to highlight the parasites. The disservice of Giemsa is that it additionally recolor other blood film elements, for example, white-bloodcells, platelets, and slide artifacts, like, dust particles.

\section{TYPES OF IMAGE \\ PREPROCESSING TECHNIQUES}

Pre-processing images usually includes eliminating lowfrequency background noise, normalizing the intensity of the distinct elements images, as well as discarding reflections and masking parts Before computational preparing we perform 'picture pre-handling' for creating information pictures. To upgrade the representation of pictures and control of datasets is the way to go image pre-handling. The development techniques can stress image artifacts, or can also head to loss of data if imperfectly used. There are several techniques commonly used once are low-pass (LPF), high-pass(HPF) and mean filtering.

\subsection{LPF}

The LPF is simplest technique for filtering. LPF is also known as "blurring" \& "smoothing" filter. The basic LPF measures the mean of a pixel as well as its all of its nearest neighbors (mask). Outcome value substitutes the original pixel value. For each image pixel this procedure is re-performed. Useful for highlighting fine features. Both non negative and negative weighted elements are present in the mask. Summation of element in the mask is zero. Useful for reducing noise and eliminating small details. Each and every pixels of intensity greater than a particular value is replaced by LPF. We apply

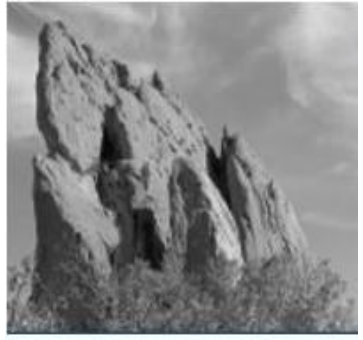

Input image

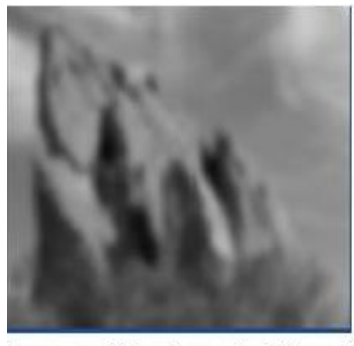

low pass filter (smoothed image)
Fig 1: Smoothed image using LPF(Low Pass Filter)

low-pass filter on the input image to smooth image as shown in Fig 1.

\subsection{HPF}

A HPF can be applied on an image to make it appear sharper. These filters push fine highlights in the image; it is only inverse of the LPF. It is a system in roughly the same path as LPF; the distinction in this filtering is the utilization of

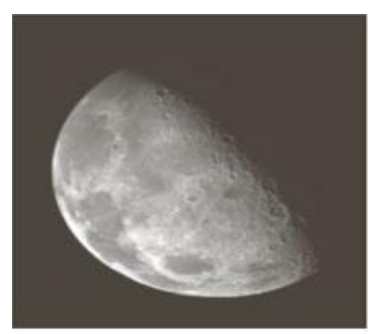

Input image

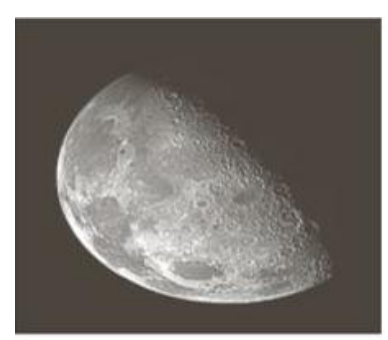

High pass filter (sharpened) image
Fig 2: Sharpened image using HPF (High Pass Filter)

convolution kernel. On the off chance that there is no variety in force, nothing happens. However, in the event that one pixel is brighter than its quick neighbors, it gets helped. It amplifies noise as shown in Fig 2. To overcome this image must not have too much noise; otherwise the noise will overpower the image. HPF just emphasizes intensive portions of the image, where the signal to-noise proportion is most noteworthy.

\subsection{Mean Filtering}

It is a straightforward, instinctive and simple to implement technique of smoothing images; it reduces the measure of brightness shift from one pixel and the following. It is for the most part used to direct noise in images. The idea of this method is just to swap the estimation of every pixel with the mean estimation of its neighbors as appeared in Fig 3, including itself. This leads in disposing of pixel qualities which are not questionable to their neighborhood. This filter is by and large expected of as a 'convolution kernel', which determines the shape and size of the surroundings to be inspected when figuring the mean as shown in Fig 4. 


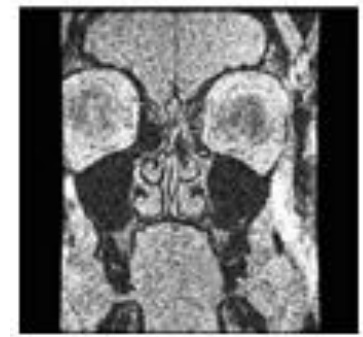

Input image

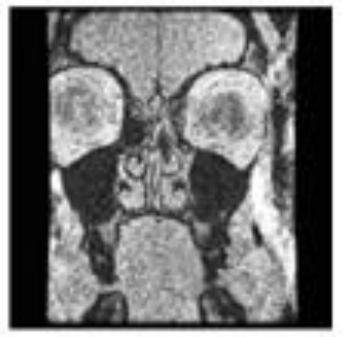

Mean Filtered image
Fig 3: Mean-Filtered Image using Mean Filtering

\begin{tabular}{|c|c|c|}
\hline$\frac{1}{9}$ & $\frac{1}{9}$ & $\frac{1}{9}$ \\
\hline$\frac{1}{9}$ & $\frac{1}{9}$ & $\frac{1}{9}$ \\
\hline$\frac{1}{9}$ & $\frac{1}{9}$ & $\frac{1}{9}$ \\
\hline
\end{tabular}

Fig 4: A 3X3 square kernel which is frequently used for smoothing

\section{TYPES OF IMAGE SEGMENTATION}

Types of Segmentation techniques are shown in Fig 5. In this technique 'digital' image is divided to form 'multiple segments'. The aim is to make the image more expressive and simpler for investigate by simplifying and altering it using segmentation. Need is when there is no direct similarity between image pixel features and the type of tissue, it is used to enhance the study of those images. It is also needed for labeling the pixels of the image.

\subsection{Thresholding segmentation:}

The 'boundaries' of the image comprising of solid elements on a contrast background is determined by thresholding technique. This procedure takes grey scale image as an input and gives a binary output by deciding on threshold ' $\mathrm{T}$ '. A singular static standard is applied to all pixels in the image concurrently.

Mathematically it is represented by, label1 as an object of the image and 0 as the background. The $\mathrm{F}(\mathrm{x}, \mathrm{y})$ is a function of thermal image, while threshold image $\mathrm{H}(\mathrm{x}, \mathrm{y})$ can be defined as follows:

$$
H(x, y)=\left\{\begin{array}{l}
1, F(x, y)>T \\
0, F(x, y)<T
\end{array}\right.
$$

\subsection{Edge Detection Segmentation}

The edge detection segmentation of an image helps in minimizing the amount of data to be processed; still it obtains important data regarding the shapes of objects in the scene. The main feature of these techniques is to get the accurate edge line with decent orientation. There are eight techniques in this segmentation; they are Roberts, Sobel, Prewitt, Kirsh, Robinson, Marr-Hildreth, LoG and Canny edge detection. Among which Marr-Hildreth, LoG and Canny edge detectors generate almost similar edge map. Canny produces exceptional output compared to all as different techniques works better under different applications.

\subsection{Region-based segmentation:}

The concept is to categorize a specific image into a several regions or classes. In which class the pixel belongs to is estimated, for every pixel of the image. There are numerous techniques and to our knowledge the performance doesn't change from one technique to the next.

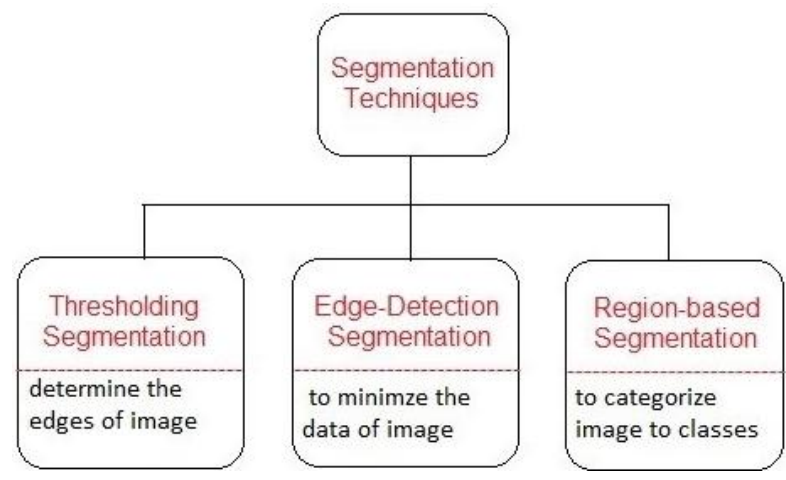

Fig 5: Types of Segmentation Techniques

\section{PROPOSED MODEL}

The architecture of the proposed model is shown in Fig 6.

\subsection{Median filtering image pre-processing}

The basic idea behind median filtering is to analyze pixel neighborhoods in image, sort their strengths Filter. It is a digital filtering method which is nonlinear and which frequently recycled to eliminate noise and which preprocessing step can be used to progress the results of processing later , It is very vastly used in image processing because it conserves edges though eliminating noise under some conditions. The idea behind this filter is to use up the signal, exchanging respective entry with the middle of nearby entries. If the window has a number of the median which is not usual is simple to define a value of the center of all entries of the window which are numerically organized. There are many such median values for an even number of inputs. This filter is a strong. Those are widely recycled as smoothtextured for processing an image, in processing of time series and signal. A major benefit of this filter is that can be excluded the result of input noise values which is large magnitudes values by the median filter. The output y of the this filter at time $t$ is included as the median of the values which is given in the input. Conforming to the moments adjacent to $t$ : where $t$ is the size of the window of the median filter.

Example. Let's say our filter size was $5 \mathrm{X} 5$, and we'll use cameraman. We get the following, with the original image, and the final image that's filtered with median filtering. This is what we expect, as median filtering is considered to smooth the image while preserving the edges too. Median filtering is specifically used for salt-and-pepper noise where it is highly possible that these noisy pixels will be shown at the beginning and at the end when sorting pixel neighborhoods, so selecting the middle value will mostly filter out these noisy values. 


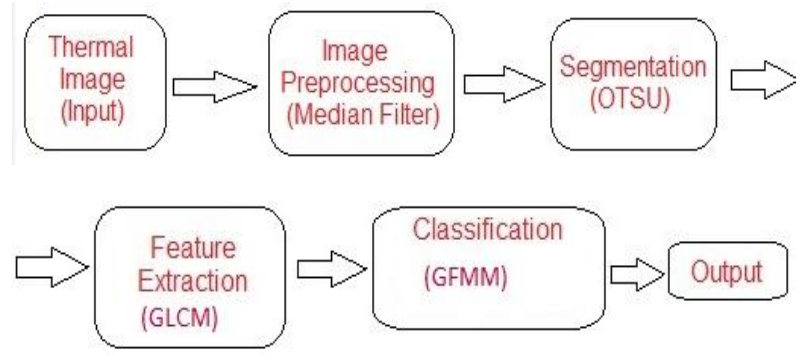

Fig 6: Architecture of Proposed Model

\subsection{Otsu image segmentation}

In Otsu image segmentation Fig 7, clustering-based image thresholding is computed automatically, or "the gray level" image is reduced to "binary" image. This procedure assumes two-modal histogram which has 2 pixel groups foreground and background pixels. Then 'optimum threshold' is computed for splitting the 2 pixel classes for minimizing or equalizing the spread, such that the inter-class alternations are maximal [16].

Mathematical representation: Let the scale of grayscale image ' $F(x, y)$ ' be ' $G=[0, L-1]$ ' and for every grayscale image the probability is assumed to be ' $P_{t}$ ' and ' $\mathrm{t}$ ' be the threshold value. Partitioning of the image is done by ' $t$ ' in 2 classes which are ' $C_{0}$ ' and ' $C_{1}$ '. Where ' $C_{0}=[0, t]$ ' and ' $C_{1}=[t+1, L-1]$

$\alpha_{0}=\sum_{i=0}^{t} P_{i}$ and $\alpha_{1}=1-\alpha$ are the probabilities of respective classes . $\mu_{0}=\sum_{i=0}^{t} \frac{i P_{i}}{\alpha_{0}}=\frac{\mu_{t}}{\alpha_{0}}$ and $\mu_{1}=\sum_{i=t+1}^{L-1} \frac{i P_{i}}{\alpha_{1}}=\frac{\mu-\mu_{1}}{1-\alpha_{0}}$ are the values of gray of respective classes . Where $\mu=\sum_{i=0}^{L-1} i P_{i}, \mu_{t}=\sum_{i=0}^{t} i P_{i}$ .Representation of 2 classes is as follows:

$\eta^{2}(t)=\alpha_{0}\left(\mu_{0}-\mu\right)^{2}+\alpha_{1}\left(\mu_{1}-\mu\right)^{2}=\alpha_{0} \alpha_{1}\left(\mu_{0}-\mu_{1}\right)^{2}$ Calculating this equation maximum value of ' $\mathrm{t}$ ' is found.

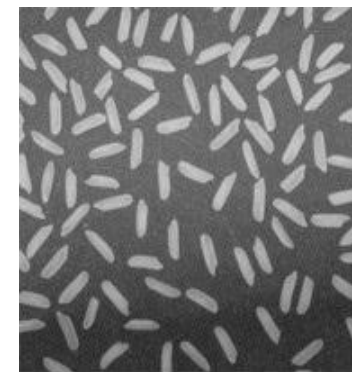

Input Image

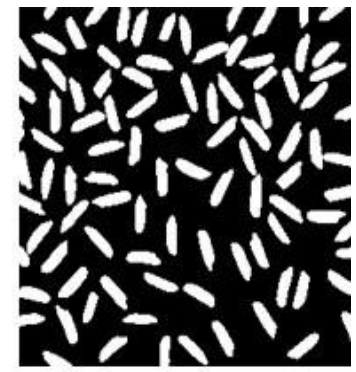

Otsu segmented output image
Fig 7: Segmented image using OTSU segmentation

\subsection{Gray Level Co-occurrence Matrix algorithm for feature extraction}

In statistical texture analysis, texture feature are calculated of detected arrangements of intensities relative to each other at particular location. Every pixel grouping are classified in three statistics, first and second order and then later in higher order depending on the sum of pixel. Second order feature extraction is performed in GLCM. . It contains a grid which holds information of dim estimations of each pixel at its relating area. The lines and segment of this grid is identical to total of dark levels. The relative recurrence between the two pixels of the component is "P $(i, j \mid d, \theta)$ ". Where'd' is the separation between pixels, " $\theta$ " is the angle, in which pixel1 intensity is " $\mathrm{i}$ " and pixel 2 intensity is ' $\mathrm{j}$.

The basic GLCM algorithm is as follow:

1. Count all sets of pixels in which the first pixel has a quality ' $i$ ', and its coordinating pair uprooted from the first pixel by $d$ has an estimation of ' $j$ '.

2. This include is entered the $i^{\text {th }}$ line and $j^{\text {th }}$ segment of the grid 'Pd[i,j]'.

3. Otsu segmented output image is: Otsu segmented output image.

4. Note that ' $\mathrm{Pd}[\mathrm{i}, \mathrm{j}]$ ' is not symmetric, since the quantity of sets of pixels having dark levels[i,j]does not as a matter of course equivalent the quantity of pixel sets having dim levels [j,i].

5. The components of ' $\mathrm{Pd}[\mathrm{i}, \mathrm{j}]$ 'can be standardized by partitioning every section by the aggregate number of pixel sets..

6. Normalized GLCM 'N[i,j]', characterized by

$$
N[i, j]=\frac{P[i, j]}{\sum_{i} \sum_{j} P[i, j]}
$$

\subsection{GFMM neural network for classification}

GFMM is an augmentation of FMM system. It utilizes minmax hyper boxes as fuzzy sets as same as unique strategies. The stars of this technique are specified underneath:

1. It performs characterization alongside clustering. It definitely builds up the efficiency in perceiving and looking the information under scrutiny.

2. With no losing of acknowledgment rate it adjusts the hyper boxes to greater hyper boxes.

3. It separates among "equal evidence" and "ignorance" by applying variations to the membership function.

4. In view of these rotations we get stable membership values".

\section{CONCLUSIONS}

In thermal image processing, we have discussed about several pre-processing techniques. But these techniques have many shortcomings. Both low-pass and high-pass might not give a step enough cut-off frequency. Mean filtering technique has the drawback of blurring the image. Median filter overcomes all these shortcomings. It removes the impulse noise and preserves the edges. This technique produces the best output image. This resultant image can be segmented by four 
techniques thresholding, edge detection and Otsu segmentation. Thresholding segmentation works well with some image but has no guarantee of object coherence that is it may have holes and extraneous pixels. In edge detection segmentation, the operation gets diffracted by some of the current edges in the noisy image. Therefore Otsu segmentation is chosen to overcome the disabilities and optimizing the process. The required part of segmented thermal image is extracted using GLCM. Similar gray level valued pixels data are maintained by GLCM in a specific locality. Then by applying GFMM we can classify the infected part and the uninfected of the cell.

\section{REFERENCES}

[1] Akther M, Ahmed K, Hasan Z. 2013 Detection of Vehicle's Number Plate at Nighttime using Iterative Threshold Segmentation (ITS) Algorithm

[2] C.A. Moxon, G.E. Grau, A.G. Craig 2011 Malaria: modification of the red blood cell and consequences in the human host

[3] C.K. Murray, R.A. Gasser Jr., A.J. Magill, R.S. Miller 2008 Update on rapid diagnostic testing for malaria

[4] C. Wongsrichanalai, M.J. Barcus, S. Muth, A. Sutamihardja, W.H. Wernsdorfer, 2008 A review of malaria diagnostic tools: microscopy and rapid diagnostic test (RDT)

[5] F. Omodeo-Salè, A. Motti, N. Basilico, S. Parapini, P. Olliaro, D. Taramelli 2003 Accelerated senescence of human erythrocytes cultured with Plasmodium falciparum

[6] F. Omodeo-Salè, A. Motti, A. Dondorp, N.J. White, D. Taramelli, 2008 Destabilisation and subsequent lysis of human erythrocytes induced by Plasmodium falciparum haemproducts

[7] Gonzalez R, Woods R. 2008 Digital Image Processing
[8] H.W. Hou, A.A.S. Bhagat, A.G.L. Chong, P. Mao, K.S.W. Tan, J. Han, C.T. Lim, 2010 Deformability based cell margination-a simple microfluidic design for malaria-infected erythrocyte separation,

[9] J.P. Shelby, J. White, K. Ganesan, P.K. Rathod, D.T. Chiu, 2003 A microfluidic model for single-cell capillary obstruction by Plasmodium falciparum-infected erythrocytes

[10] J.P. Mills, M. Diez-Silva, D.J. Quinn, M. Dao, M.J. Lang, K.S.W. Tan, C.T. Lim, G. Milon, P.H. David, O. Mercereau-Puijalon, S. Bonnefoy, S. Suresh, 2007 Effect of plasmodial RESA protein on deformability of human red blood cells harbouring Plasmodium falciparum

[11] L. Gervais, N. de Rooij, E. Delamarche, 2011 Microfluidic chips for point-of-care immunodiagnostics

[12] M. Sezgin and B. Sankur 2004 Survey over image thresholding techniques and quantitative performance evaluation

[13] Nobuyuki Otsu 1979 A threshold selection method from gray-level histograms

[14] Ostu N A. 1979 Threshold Selection Method from GrayLevel Histograms

[15] . Lima, T. Ishikawa, Y. Imai, M. Takeda, S. Wada, T. Yamaguchi 2008 Radial dispersion of red blood cells in blood flowing through glass capillaries: the role of hematocrit and geometry

[16] R. Fan, O. Vermesh, A. Srivastava, B.K.H. Yen, L. Qin, H. Ahmad, G.A. Kwong, C.- C. Liu, J. Gould, L. Hood, J.R. Heath 2008 Integrated barcode chips for rapid, multiplexed analysis of proteins in microliter quantities of blood

[17] Sujji G, Lakshmi YVS, Wiselin G. 2013 MRI Brain Image Segmentation based on Thresholding. International Journal of Advanced Computer Research 